\title{
Wavelet Based Change Detection for Four Dimensional Assimilation Data in Space and Time Domains
}

\author{
Kohei Arai $^{1}$ \\ Graduate School of Science and Engineering \\ Saga University \\ Saga City, Japan
}

\begin{abstract}
Method for time change detection of four dimensional assimilation data by means of wavelet analysis is proposed together with spatial change detection with satellite imagery data. The method is validated with assimilation data and the same scale of satellite imagery data. Experimental results show that the proposed method does work well in visually.
\end{abstract}

Keywords-Assimilation; change detection; wavelet analysis.

\section{INTRODUCTION}

Data assimilation is useful for numerical prediction of global issues including global warming, weather forecasting, and climate changes [1]-[3]. In particular, four dimensional assimilations, three dimensional space and time dimension, space and time domains, is useful. There are some problems on the current four dimensional assimilations as follows,

(1)Prediction accuracy is not good enough,

(2)Precision of input data is not good enough,

(3)Not so stable solution can be derived

(4)Boundary conditions cannot be given properly,

(5)Change detection performance is not good enough.

In order to overcome the aforementioned problems, (3) and (5), wavelet analysis is introduced here in this paper. Namely, wavelet analysis based prediction is attempted for getting stable solutions. Also, wavelet analysis based space and time change detections are attempted as well.

One of the examples is shown in this paper. Time series of three dimensional air temperature and relative humidity are created with assimilation model of which earth observation satellite data derived profiles. These are called as "state variables". Space and time changes on the state variables are detected in terms of the change locations and change amount. Then extremely hot summer in Japan in 2010 is predicted. It is mainly caused by the jet stream winding in northern hemisphere and the location and magnitude of high pressure system situated in the Pacific Ocean areas. Such this result could be come out from the proposed method.
The following section describes the research background together with the proposed wavelet based method followed by experiments conducted. Then conclusion with some discussions is followed.

\section{PROPOSED METHOD}

\section{A. Four Dimensional Assimilation}

Three dimensional box type of space is assumed for each district in assimilation. In the space, remaining variables, mass, energy and angular momentum are maintained and balanced. Such remaining variable $\mathrm{X}$ changed in time domain, $\Delta \mathrm{X} / \Delta \mathrm{t}$, and is same as supplied variables from the top (Ftop), from the surface (Fsfc), and from the horizontal directions (Fside) as shown in Figure 1.

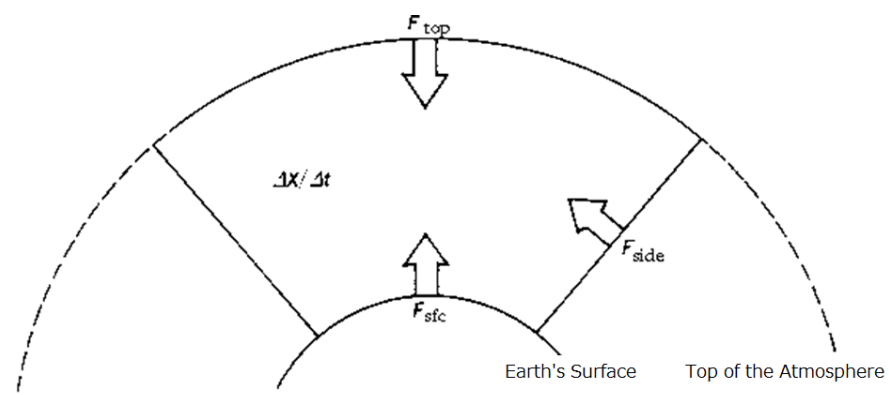

$\Delta X^{\prime} \Delta t:$ Time changes of remained variables

$\boldsymbol{F}_{\text {top }}:$ Suppied net flux energy from top of the atmosphere

$\boldsymbol{F}_{\text {side }}$ : suppied net flux energy from horizontal direction

$\boldsymbol{F}$ sfc : Suppied net flux energy from the earth's surface

Figure 1 Illustrative view of the assimilation model

The space is divided with meshes in vertical and horizontal directions as shown in Figure 2. In the meshes, variables are maintained and balanced. For instance, input energy to the mesh is totally equal to output energy from the mesh. Thus partial differential equation can be formulated. Because partial differentiation in space is partial differentiation in time, then prediction can be done. Therefore, assimilation can be done. This is called assimilation model. 


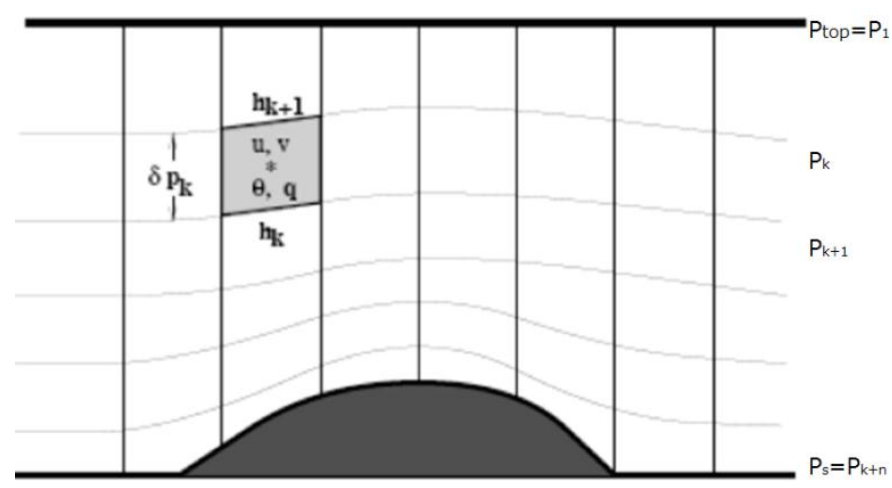

Figure 2 Assimilation model with meshed space and time function of variables.

Variables, input data for assimilation model are obtained from the earth observation satellite data, in particular, infrared sounder as well as microwave sounder data. Table 1 and 2 shows example of the input data, variables, for assimilation model derived from the specific sensor data.

Table 1 Example of the variables, input data for assimilation model and data sources mainly from the satellite data.

\begin{tabular}{|l|l|}
\hline Conventional Data & \multicolumn{2}{l|}{} \\
\hline Radiosondes & $\mathrm{u}, \mathrm{v}, \mathrm{T}, \mathrm{q}, \mathrm{P}_{\mathrm{s}}$ \\
\hline PIBAL winds & $\mathrm{u}, \mathrm{v}$ \\
\hline Wind profiles & $\mathrm{u}, \mathrm{v}$ \\
\hline Conventional, ASDAR, and MDCRS aircraft reports & $\mathrm{u}, \mathrm{v}, \mathrm{T}$ \\
\hline NEXRAD radar winds & $\mathrm{u}, \mathrm{v}$ \\
\hline Dropsondes & $\mathrm{u}, \mathrm{v}, \mathrm{T}, \mathrm{P}_{\mathrm{s}}$ \\
\hline PAOB & $\mathrm{P}_{\mathrm{s}}$ \\
\hline GMS, Meteosat, cloud drift IR and visible winds & $\mathrm{u}, \mathrm{v}$ \\
\hline MODIS clear sky and water vapor winds & $\mathrm{u}, \mathrm{v}$ \\
\hline GOES cloud drift IR winds & $\mathrm{u}, \mathrm{v}$ \\
\hline GOES water vapor cloud top winds & $\mathrm{u}, \mathrm{v}$ \\
\hline Surface land observ ations & $\mathrm{P}_{\mathrm{s}}$ \\
\hline Surface ship and buoy observ ations & $\mathrm{u}, \mathrm{v}, \mathrm{T}, \mathrm{q}, \mathrm{P}_{s}$ \\
\hline SSSM/I & Rain rate, wind speed \\
\hline TMI & Rain rate \\
\hline QuikSCAT & $\mathrm{u}, \mathrm{v}$ \\
\hline
\end{tabular}

Table 2 Example of sensor name and satellite name of the data sources for assimilation model.

\begin{tabular}{|c|c|}
\hline \multicolumn{2}{|l|}{ Satellite Data } \\
\hline TOVS 1b Radiances & $\begin{array}{l}\text { AMSU-A: N15, N16, N18 } \\
\text { AMSU-B: N15, N16, N17 } \\
\text { MHS: N18 } \\
\text { HRRS2: TIROS-N, N6, N7, N8, N9, } \\
\text { N10, N11, N12, N14 } \\
\text { HIRS3: N16, N17 } \\
\text { HRRS4: N18 } \\
\text { MSU: TIROS-N, N6, N7, N8, N9, } \\
\text { N10, N11, N12, N14 } \\
\text { SSU: TIROS-N, N6, N7, N8, N9, } \\
\text { N10, N11, N14 }\end{array}$ \\
\hline EOS/Aqua Level 1b Radiances & AIR.S (150 channels), AMSU-A \\
\hline SSM $/$ I radiances & $\begin{array}{l}\text { DMSP-8, DMSP-10, DMSP-11, } \\
\text { DMSP-13, DMSP-14, DMSP-15 (7 } \\
\text { channels) }\end{array}$ \\
\hline GOES sounder $T_{B}$ & $\begin{array}{l}\text { GOES-08, GOES-10, GOES-12 } \\
\text { Channels } 1 \text { - } 18\end{array}$ \\
\hline SBUV2 ozone (Version 8 retriev als) & Nimbus 7, NOAA 9, 11, 14, 16, 17 \\
\hline
\end{tabular}

The variables are formed time series of spatially aligned three dimensional geophysical data as shown in Figure 3. Layered two dimensional geophysical data, atmospheric pressure, air temperature, relative humidity, etc. aligned along with time direction. Using assimilation model with satellite data derived geophysical data as input data, such this four dimensional geophysical data are created.

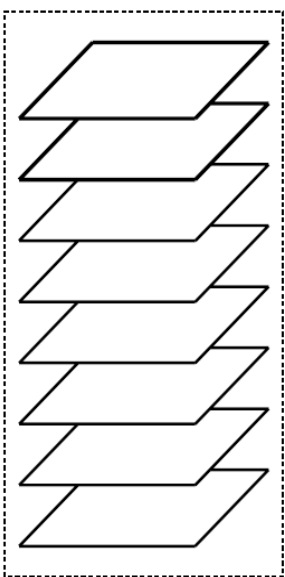

$t-1$

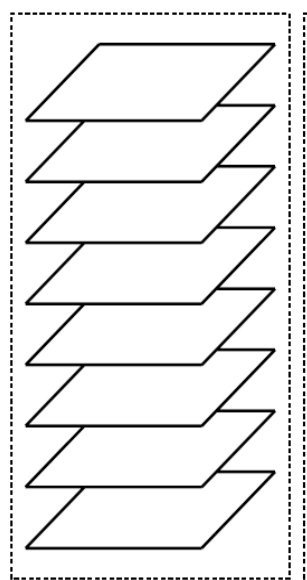

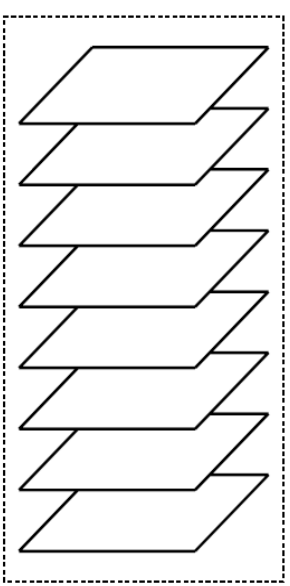

$\mathrm{t}+1$
Figure 3 Four dimensional assimilation data

\section{B. Wavelet Analysis Based Problem Solving Method and Change Detections in Space and Time Domains}

One dimensional wavelet transformation can be expressed in equation (1).

$\mathrm{F}=\mathrm{Wf}$

where $f$ denotes geophysical data in space or time domain while $W$ denotes wavelet transformation matrix. Since, $\left[G_{i j k}\right]^{t}$ $=G_{k j i}$, then three dimensional wavelet transformation can be written as follows,

$$
\mathrm{F}=\left[\mathrm{W}_{\mathrm{k}}\left[\mathrm{W}_{\mathrm{j}}\left[\mathrm{W}_{\mathrm{i}} \mathrm{G}_{\mathrm{ijk}}\right]^{\mathrm{t}}\right]^{\mathrm{t}}\right]^{\mathrm{t}}
$$

Not only three dimensional wavelet transformation, but also $\mathrm{n}$ dimensional wavelet transformation can be expressed as follows,

$$
\mathrm{F}=\left[\mathrm{W}_{\mathrm{n}}\left[\mathrm{W}_{\mathrm{n}-1}\left[\ldots\left[\mathrm{W}_{1} \mathrm{G}_{12 \ldots \mathrm{n}}\right]^{\mathrm{t}}\right]^{\mathrm{t} . .}\right]^{\mathrm{t}}\right.
$$

This wavelet transformation is called as decompositions.

One of the specific features of the wavelet transformation is that the original geophysical data $G$ can be reconstructed with the calculated wavelet frequency components, $F$, perfectly. This reconstruction processes is called inverse wavelet transformation.

Also, wavelet analysis is useful for solving integral equations as well as partial differential equation [ ]. Therefore, wavelet analysis based method can be used for solving the partial differential equations in the assimilation model.

Furthermore, wavelet analysis based Multi Resolution Analysis: MRA is used for change detections in space and time domains. Using MRA, wavelet frequency components are calculated. If the reconstruction is applied to the frequency components without low frequency component, then spatial and time changes are extracted because the reconstruction is made with high frequency components only. 


\section{Proposed MRA Based Change Detection Method}

Change detection performance can be evaluated as follows,

$$
\begin{aligned}
J 1(\alpha)= & \sqrt{\frac{\sum_{t} \sum_{z} \sum_{y} \sum_{x}\left(d_{\alpha}^{*}(x, y, z, t)-d(x, y, z, t)\right)^{2}}{X \times Y \times Z \times T I M E}} \\
& d(x, y, z, t): \text { Observation Data } \\
& d_{\alpha}^{*}(x, y, z, t): \text { Reconstructed Data (CR=alpha) } \\
& X, Y, Z, T I M E: \text { Amount of Data } \\
& X=32, Y=32, Z=8, T I M E=12
\end{aligned}
$$

where $\mathrm{J} 1$ denote cost function which represent change detection performance. Namely, Root Mean Square: RMS difference between the original and the reconstructed images is a good measure for evaluation of change detection performance. The procedure of the proposed method is as follows,

(1) Wavelet transformation is applied to the original image,

(2) Wavelet frequency component, coefficients are sorted in accordance with the coefficient values,

(3) Remove the first $\mathrm{n}$ coefficients form the minimum

(4) Reconstruct image with the rest of coefficients of component

(5) Determine an optimum $\mathrm{n}$ of which the changes of RMS difference is saturated

\section{EXPERIMENTS}

\section{A. Change Detection Using Subtraction}

One of the examples of the change detection in space and time domains is shown. That is cloud movement analysis with Geostationary Meteorological Satellite: GMS imagery data. Figure 4 shows (a) original time series of GMS imagery data, (b) binalized images, and (3) detected changes in space and time domains by using subtraction between adjacent binalized images Also change detection and object movement analysis can be done with optical flow model as well. Thus the clouds movement can be analyzed. It is sensitive to the threshold processes. Namely, detected changes depend on binalized results.

\section{B. Change Detection of Air Temperaure and Relative Humidity Profiles in Space and Time Domains Using the Aforementioned Proposed MRA Based Method}

One of examples of change detections based on the proposed wavelet MRA analysis based method is shown. Figure 5 shows monthly average of the relative humidity profiles which were acquired in February and August in 1992. The profile can be formed with 8 layers in altitude direction, $\mathrm{z}$, and with 1 degree resolution in horizontal directions, $\mathrm{x}$ and $\mathrm{y}$. Also the relative humidity ranges from 0.005 to $20 \mathrm{~g} / \mathrm{Kg}$.

Change detection in time domain can be done with the reconstruction with all wavelet frequency components except LLLL component. LLLL denotes low frequency components of $\mathrm{x}, \mathrm{y}, \mathrm{z}$, and time directions. However, it is quite obvious that the resultant image shows detected changes in space and time domains as shown in Figure 5 (c).
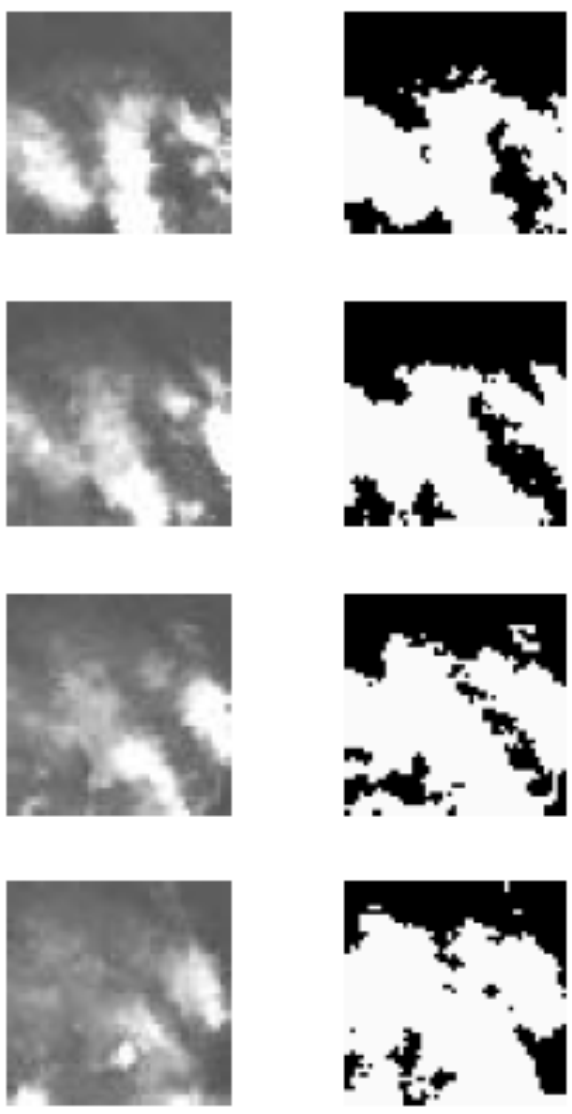

(a)Original

(b)Binalized

(c)Changes

Figure 4 Example of change detection in space and time domains

Also, the resultant image seems that not so good performance in terms of detected changes in the image. Then reconstruction with all wavelet frequency components except LLLL component together with the first $\mathrm{n}$ wavelet coefficients is tried. In this connection, $100 \%-\alpha \%$ of wavelet coefficients are removed. $\alpha$ corresponds to data compression ratio.
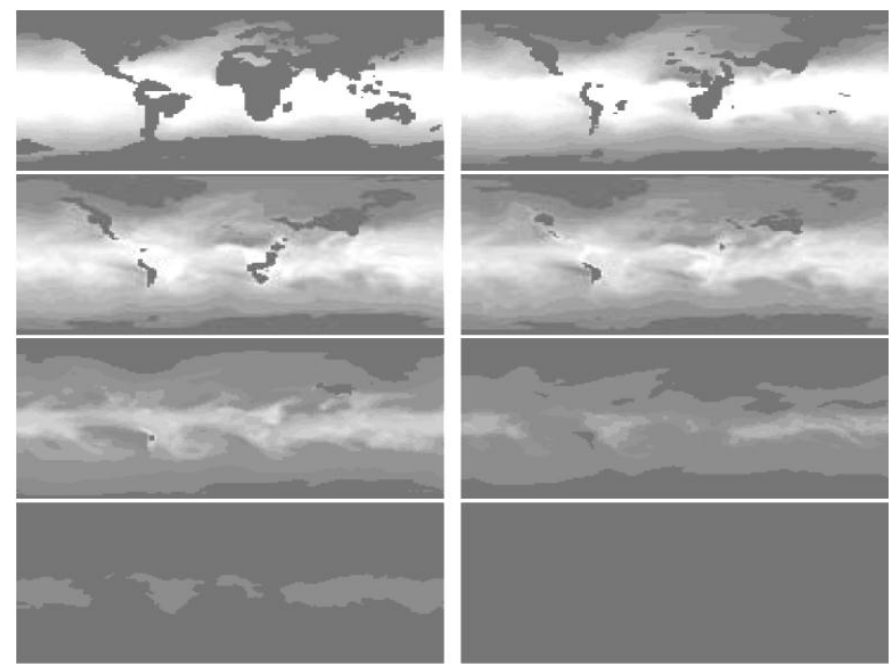

(a)Relative humidity in August 1992 

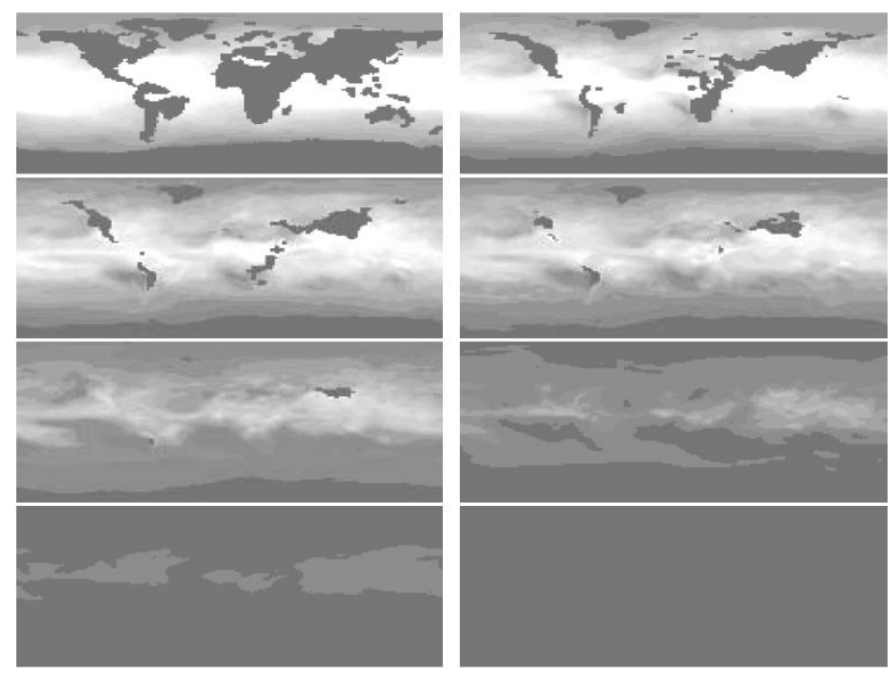

(b)Relative humidity in February 1992
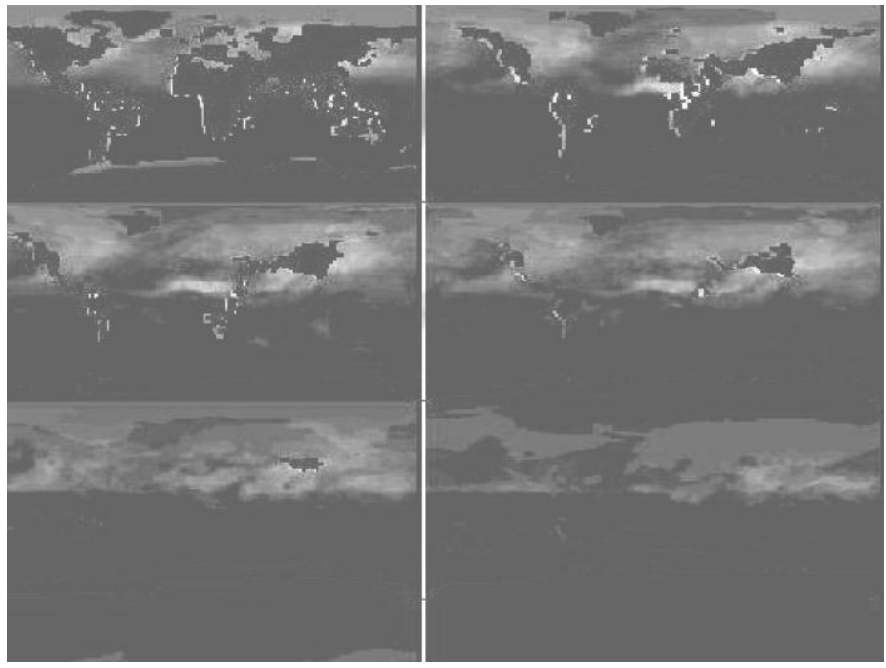

(c)Detected changes with all frequency components except LLLL component

Figure 5 Detected changes by means of the MRA based method, reconstruction of original image without LLLL component

\section{Evaluation of Data Compression Ratio}

As the aforementioned in the previous section, change detection performance is highly correlated with the RMS difference between the original and the reconstructed images. Therefore, change detection performance for the proposed method is evaluated with calculation of RMS difference as a function of data compression ratio.

Almost homogeneous ocean area is selected for the evaluation. In this Pacific Ocean area which is shown in Figure 6, relative humidity is relatively homogeneous. Relation between RMS difference and data compression ratio is shown in Figure 7. Although RMS error for the data compression ratio of $100 \%$ (which means no data compression is applied) is obviously zero, it is getting large in accordance with decreasing of data compression ratio. For instance, RMS error for data compression ratio of $10 \%$ is 0.4 . This implies that 0.4 of RMS error has to be accepted for $1 / 10$ of data compression.

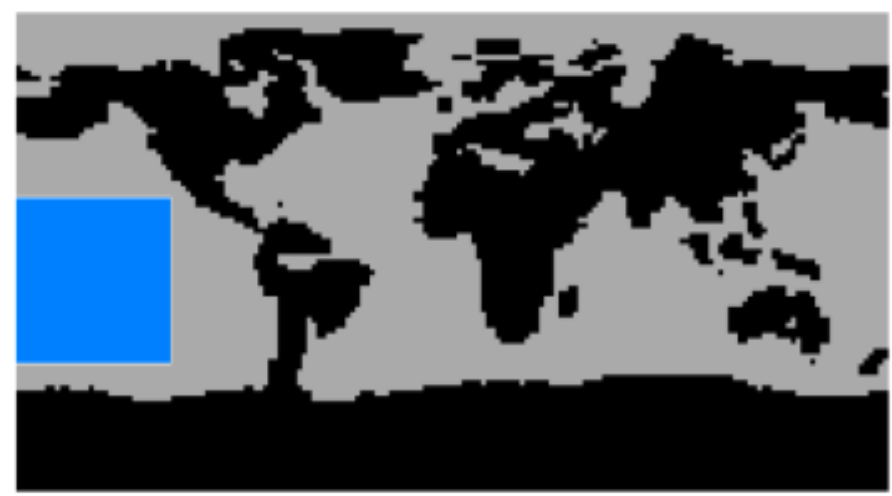

Figure 6 Intensive study area for RMS difference evaluations

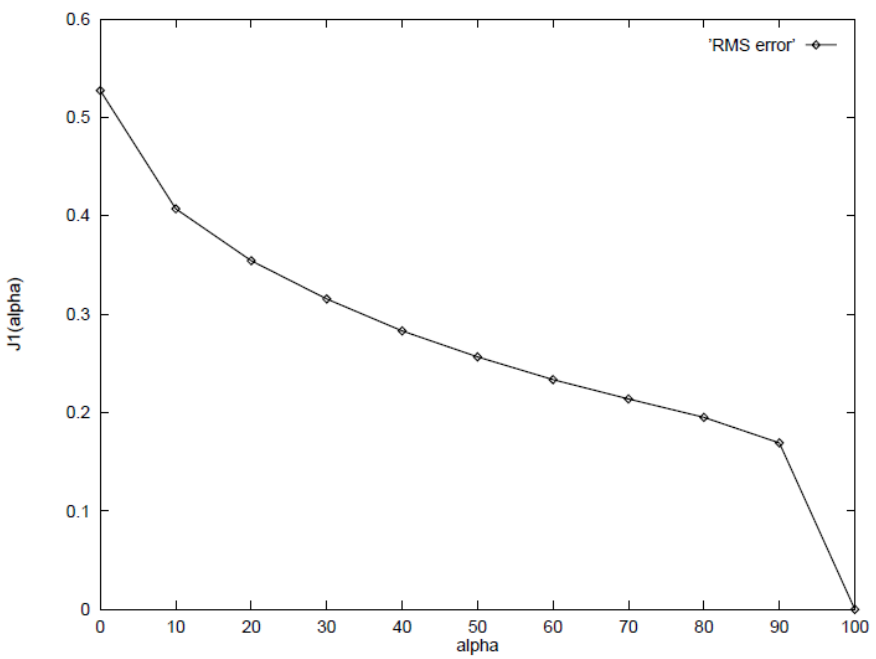

Figure 7 Relation between RMS difference and data compression ratio

\section{CONCLUSION}

Method for time change detection of four dimensional assimilation data by means of wavelet analysis is proposed together with spatial change detection with satellite imagery data. The method is validated with assimilation data and the same scale of satellite imagery data. Experimental results show that the proposed method does work well in visually. Also the relation between RMS error and data compression ratio is clarified.

\section{ACKNOWLEDGMENT}

The author would like to thank Dr. Kaname Seto for his effort to conduct experiments.

\section{REFERENCES}

[1] R. Daley, Atmospheric data analysis, Cambridge University Press, 1991.J. Clerk Maxwell, A Treatise on Electricity and Magnetism, 3rd ed., vol. 2. Oxford: Clarendon, 1892, pp.68-73.

[2] Ide, K., P. Courtier, M. Ghil, and A. C. Lorenc (1997) Unified Notation for Data Assimilation: Operational, Sequential and Variational Journal of the Meteorologcial Society of Japan, vol. 75, No. 1B, pp. 181-189

[3] John M. LEWIS; S. Lakshmivarahan, Sudarshan Dhall, "Dynamic Data Assimilation : A Least Squares Approach", Encyclopedia of Mathematics and its Applications 104, Cambridge University Press, 2006 


\section{AUTHORS PROFILE}

Kohei Arai, He received BS, MS and PhD degrees in 1972, 1974 and 1982, respectively. He was with The Institute for Industrial Science, and Technology of the University of Tokyo from 1974 to 1978 also was with National Space Development Agency of Japan (current JAXA) from 1979 to 1990. During from 1985 to 1987, he was with Canada Centre for Remote Sensing as a Post Doctoral Fellow of National Science and Engineering Research Council of Canada. He was appointed professor at Department of Information Science,
Saga University in 1990. He was appointed councilor for the Aeronautics and Space related to the Technology Committee of the Ministry of Science and Technology during from 1998 to 2000 . He was also appointed councilor of Saga University from 2002 and 2003 followed by an executive councilor of the Remote Sensing Society of Japan for 2003 to 2005 . He is an adjunct professor of University of Arizona, USA since 1998. He also was appointed vice chairman of the Commission "A" of ICSU/COSPAR in 2008. He wrote 30 books and published 332 journal papers. 